\title{
Does motor deficit in children with cerebral palsy correlate with diffusion tensor metrics abnormalities in thalamocortical pathways?
}

\author{
Amal Mohammed Mahanna ${ }^{\text {* }}$, Nahed Abd El-Gaber El-Toukhy², Amani Ezzat Mousa², Khaled Fathy Megahed ${ }^{3}$ and
} Germeen Albair Ashamallah²

\begin{abstract}
Background: Cerebral palsy (CP) is a group of motor-impairment signs secondary to many disorders that interfere with early brain development and are usually related to white matter injury in children. Most studies are focused on the study of motor tracts, mainly the corticospinal tract (CST). Diffusion tensor imaging (DTI) is a reliable imaging modality providing an appropriate method of detection of white matter microstructure abnormalities. The purpose of this study is to investigate the relationship between DTI observed motor CST injury, sensory pathways (thalamic radiations) injury, and motor functions.

Results: This study shows significant involvement (reduction in DTI fiber count) of the superior thalamic radiation (in severe cases) with atrophy of the anterior thalamic radiations (ATR) or posterior thalamic radiations (PTR) in most cases with a significant reduction in fractional anisotropy (FA) and elevation in mean diffusivity (MD) values. In addition, the degree of motor affection shows a significant negative correlation with FA and a significant positive correlation with MD values. Diffusion tensor imaging shows a significant reduction in FA within the examined tracts between CP and control at the Rt CST, Lt CST, Rt corticothalamic radiation (CTR), and Lt CTR with significant cutoff values of $\leq 0.449, \leq 0.472, \leq 0.432$, and $\leq 0.44$, respectively.

Conclusions: This study demonstrates disruption of thalamocortical and corticospinal tracts in CP patients, which reflects that both sensory and motor tract affection have a valuable role in the pathophysiology of motor dysfunction in CP patients.
\end{abstract}

Keywords: Cerebral palsy, Diffusion tensor imaging, Diffusion tensor tractography, Gross motor function, Motor pathway, Sensory pathway

\section{Background}

Population-based studies revealed that the incidence of $\mathrm{CP}$ range from 1.5 to 5 per 1000 children of a defined age range [1]. The overall birth incidence of $\mathrm{CP}$ is about 2 per 1000 live births, and it is considered the commonest etiology of disability in children [2].

The term CP describes a variable group of conditions comprising irreversible non-progressive central motor dysfunction, which lead to impairment in muscle tone,

\footnotetext{
* Correspondence: Amal_Mahanna@hotmail.com

${ }^{1}$ Diagnostic and Interventional Radiology, Ministry of Health, Cairo, Egypt

Full list of author information is available at the end of the article
}

movement, and posture. Multiple other signs are frequently associated with the primary motor abnormalities, such as altered sensation, communication, behavioral difficulties, intellectual disability, seizures, and musculoskeletal problems [3].

Periventricular leukomalacia (PVL) is supposed to be the most common neuropathological condition in premature infants and the primary etiology of $\mathrm{CP}$ development [4].

From whole white matter tracts, the pyramidal tract and thalamic radiations undergo early maturation. Throughout the process of brain maturation, these two 
essential white matter pathways are susceptible to injuries that lead to neurologic affections, with subsequent development of the typical manifestations of bilateral (either diplegia or quadriplegia) spastic CP in children born preterm [5].

Brain imaging researches via conventional magnetic resonance imaging (MRI) have demonstrated that $80 \%$ of affected children have abnormal brain structures [6].

Despite conventional magnetic resonance imaging has a role in recognition of WM affection, it does not give data about the extent of specific WM tracts injury. Diffusion tensor imaging (DTI) detects restrictions in the random water molecules movement by different tissue barriers as myelin and can be used to reconstruct affected WM tracts [7].

DTI estimation of fractional anisotropy (FA) values in different brain areas can provide quantitative as well as qualitative information about the white matter tracts, and could evaluate abnormalities in white matter, and also can provide useful data in terms of white matter repair. Nowadays, DTI is considered a helpful tool in evaluating white matter injury, providing precise data about the disease severity and prognosis [8].

In this study, we assess the average values of fractional anisotropy (FA) and mean diffusivity (MD), as well as fiber count in CST and thalamic radiations in children diagnosed as CP with age/sex-matched controls, aiming to determine if both motor and sensory tract injury would show correlation with clinical motor grades assessed by Gross Motor Function Classification System (GMFCS).

\section{Methods}

Informed consent was obtained from the parents according to our Institutional Review Board.

\section{Patients}

This prospective case-control study was done between 2017 and 2020 on 96 children; of them, 70 were diagnosed clinically as $\mathrm{CP}$ patients, and 26 were the control group. A total of 70 patients with CP, 46 males (65.7\%) and 24 females (34.3\%), were recruited; their ages ranged from 2 to 15 years. Enrollment criteria were as follows: children aged more than 2 years; they are diagnosed clinically as $\mathrm{CP}$ patients; they required brain MRI either for diagnosis or follow-up. Exclusion criteria were as follows: children with myelodysplasias and neuromuscular disorders; children with cochlear implants or cardiac pacemaker. The control group included ten girls (38.5\%) and 16 boys $(61.5 \%)$; their ages ranged from 2 to 15 years.

\section{Assessments}

Clinical assessment was done with the detailed perinatal history of preterm labor, caesarian section delivery, low birth weight, birth hypoxia, and neonatal intensive care unit (NICU) admission; motor neurological examination by a pediatric neurologist was also recorded and involved CP type (either diplegia, quadriplegia or hemiplegia), and best motor skill achieved by patients using GMFCS.

\section{MRI acquisition protocol}

MRI study with standard sequences (axial T1/T2 \& FLAIR) precede the DTI protocol, done on a $1.5-\mathrm{T}$ whole-body scanner (Ingenia; Philips Healthcare Medical Systems, Best, Netherlands) using head-neck coil with the head maintained in a supine position.

All patients were sedated using 10\% Chloral hydrate. It was given in a dose of $0.25-0.50 \mathrm{ml} / \mathrm{kg}$ orally or by nasal feeding; then, patients were subjected to a scan after sleeping soundly. No patient was in need of general anesthesia.

\section{(A) Conventional MRI sequences}

All imaging was performed in the axial plane with the following parameters: $5 \mathrm{~mm}$ slice thickness, $0.5 \mathrm{~cm}$ interslice gap, FOV $250 \times 170 \mathrm{~mm}^{2}$, and matrix $80 \times$ 80. Routine sequences with the following parameters were used: T1-weighted sequences (TR $450 \mathrm{~ms}$, TE $15 \mathrm{~ms}$ ); T2-weighted sequences (TR $3960 \mathrm{~ms}$, TE 110 $\mathrm{ms}$ ); FLAIR (TR $6000 \mathrm{~ms}$ and TE $120 \mathrm{~ms}$ and TI $1800 \mathrm{~ms})$.

\section{(B) Diffusion tensor imaging (DTI)}

It was acquired after the routine sequences using single-shot EPI sequence with the sensitivity-encoding, or SENSE, parallel-imaging scheme (reduction factor, 2) (repetition time: $7300 \mathrm{~ms}$; echo time: $75 \mathrm{~ms}$ ), with a diffusion-encoding gradient in 15 independent orientations, imaging matrix $128 \times 128$, FOV of $230 \mathrm{~mm}, b$ value of $1000 \mathrm{~mm}^{2} / \mathrm{s}$, and number of acquisitions = two. Transverse sections of $2.75 \mathrm{~mm}$ thickness were obtained parallel to the anterior commissure-posterior commissure line. Fifteen sections enclosed the whole hemisphere and brainstem with no gaps. The total imaging time range from 10 to $25 \mathrm{~min}$ according to the sequences added to the routine MR imaging examinations.

\section{(III)Diffusion tensor tractography (DTT)}

Reconstruction of specific WM tracts was conducted by streamline tractography by utilizing fiber assignment 
continuous tracking algorithm (FACT). It is mainly reliant on anisotropy direction and proceeds from an initially determined point in the direction of the principal eigenvector from voxel to voxel. Multiple ROIs were manually defined by freehand drawing and assigned to the specific tract anatomical course. The termination criteria used for fiber tracking comprised an FA threshold of $<0.2$ and an angle change between the ellipsoids of $>45^{\circ}$, at which points the fiber path is terminated.

Multiple ROIs were utilized for a tract of interest, with two ROI types; inclusion (and) ROI and exclusion (not) ROI, the choice of which depended on the distinctive trajectory of each tract. WM fibers that penetrate the manually defined inclusion ROIs with dissection of those penetrating the exclusion ROIs were assigned to the specific tracts. Fibers outside the tracts of interests were discarded by utilizing the "NOT" operation.

\section{MRI data processing and analyses}

DTI imaging data was transferred to an offline workstation (extended workspace "EWS") (Release 2.5.3.0; Dell, Round Rock, Tex); Pride software (Philips Medical Systems). The following maps were obtained: FA maps, directionally encoded color FA maps, 3D fiber tractography maps.

\section{(A) Qualitative analyses}

\section{(1) 2D analyses}

Anisotropy values are calculated for each voxel, and FA uncolored and colored maps are created, where color scheme (red-green-blue colors) reflects tract direction (i.e., the red color representing fibers with right-left orientation; green representing antero-posterior direction; and blue color for cranio-caudal orientation) and color brightness/hue represents diffusion anisotropy (FA) value.

\section{(2) 3D analyses $\Rightarrow$ Diffusion tensor tractography (DTT)}

The 3D track reconstruction was carried out by utilizing the FACT approach.

\section{Reconstruction of tracts Thalamic radiations}

To reconstruct the thalamic radiations, anterior thalamic radiation: the first $\mathrm{ROI}$ was placed in the axial slice (thalamus), and the second ROI was placed in the coronal slice (frontal lobe); superior thalamic radiation: the first ROI was placed in the axial slice (thalamus), while the second ROI was placed over (frontal and parietal lobes) in the axial slice; posterior thalamic radiation: the first $\mathrm{ROI}$ was positioned in the axial slice (thalamus), and the second ROI was positioned in the coronal slice (occipital lobe).

\section{Corticospinal tract (CST)}

The CST arises from the precentral motor cortex and descends via the corona radiata and posterior $1 / 3$ of the PLIC to the pons and caudally the medulla and spinal cord. The first ROI is positioned on the color map over the anterior pons. The second ROI is positioned ipsilateral over the motor cortex.

\section{(B) Quantitative analyses (estimation of DTI metrics)}

DTI metrics including FA, MD (ADC values) for CST, and CTRs were automatically computed for the entire reconstructed tract, and the average values for each tract were calculated. After that, the number of fibers was measured in all depicted tracts individually. Such measurements are considered as a type of DTT parameter, which means a quantitative measure of connectivity between anatomical locations as detected by the ROIs.

For this study, the analysis was focused on thalamocortical pathways and corticospinal tracts. We suppose that the corticothalamic radiations (transmitting sensory impulses) and the corticospinal tracts (transmitting motor orders) are responsible for the clinical picture of CP patients.

\section{Statistical analysis}

Statistical analysis was carried out by utilizing IBM SPSS (Statistical Package for Social Science) Corp. Released 2013. IBM SPSS Statistics for Windows, Version 20. Armonk, NY: IBM Corp. Qualitative data were defined by utilizing number and percent, while quantitative ones were defined by utilizing median (minimum and maximum) for non-parametric data and mean, and the standard deviation for parametric data following testing normality using Kolmogorov-Smirnov test. Analysis of ranked variables was performed using Spearman's rank correlation test. A significance level of 0.05 was utilized for all the previously utilized tests.

\section{Results}

\section{Descriptive results and clinical features}

In this study, 96 children were scanned (Table 1): 70 of them were diagnosed clinically as CP, they included 46 males $(65.7 \%)$ and 24 females (34.3\%) with an average age of 3 years (range $2-15$ years). The other 26 children constitute the control group (including ten girls (38.5\%) and 16 boys $(61.5 \%)$ with a mean age of 5 years (range 
Table 1 Demographic characters of the studied cases

\begin{tabular}{|c|c|c|c|c|c|}
\hline \multirow[t]{2}{*}{ Variables } & \multicolumn{2}{|c|}{ CP group $(n=70)$} & \multicolumn{2}{|c|}{ Control group $(n=26)$} & \multirow{2}{*}{$\begin{array}{l}\text { Test of } \\
\text { significance }\end{array}$} \\
\hline & No & $\%$ & No & $\%$ & \\
\hline \multicolumn{5}{|l|}{ Sex } & \multirow{3}{*}{$\begin{array}{l}{ }^{2}=0.145 \\
p=0.704\end{array}$} \\
\hline Male & 46 & $65.7 \%$ & 16 & $61.5 \%$ & \\
\hline Female & 24 & $34.2 \%$ & 10 & $38.4 \%$ & \\
\hline \multicolumn{5}{|l|}{ Age/years } & \multirow{3}{*}{$\begin{array}{l}z=1.79 \\
p=0.073\end{array}$} \\
\hline Mean & 3 & & 5 & & \\
\hline Range & $2-15$ & & $2-12$ & & \\
\hline
\end{tabular}

2-12 years). The difference in age and sex among both groups was not significant $(p>0.05)$.

The CP group included 46 (65.7\%) preterm children and $24(34.3 \%)$ term children. The mean gestational weight was $2812.86 \pm 503.52$ (range, $1600-3800 \mathrm{~g}$ ). The gestational age in infants with CP was less than those in controls. The birth weight of children with CP (2812.86 \pm 503.52) was significantly lower than birth weight among the control group $(3161.54 \pm 181.28)(p=0.001)$. In total, $26(37.1 \%)$ of children were suffering from perinatal hypoxia in the CP group, whereas 36 (51.4\%) were admitted to the intensive care infant unit following delivery.

In terms of gross motor functions, minimal or moderate affection (classes I, II, and III at GMFCS) was reported in 30 cases $(42.85 \%)$, whereas severe affection (classes IV and V at GMFCS) was reported in 40 (57.14\%).

\section{The pattern of damage at conventional MR imaging}

In the $\mathrm{CP}$ group, abnormalities were observed in 54 children (77.1\%) on conventional imaging. In 16 children (22.9 \%) of the CP group, conventional MRI imaging could not find abnormalities compatible with motor affection.

Most of the studied patients had periventricular white matter injury (57.1\%), followed by grey matter damage (8.6\%), malformations (5.7\%), and miscellaneous disorders $(2.9 \%)$. In terms of MRI results, $22.9 \%$ of the studied CP patients and all control groups showed normal conventional MRI studies.

\section{DTI metrics analysis}

Group comparison of FA values among CP cases and controls revealed lower FA values within CST and CTRs in the patient group.

Tables 2 shows mean FA and MD measurements in each tract compared among two groups. There is an overall tendency of decreased FA in the CP groups compared to FA in the control group with statistically significant $p$ values $(p<0.001)$.

\section{Correlation among DTI metrics and clinical motor grade}

A significant negative association among clinical motor grade assessed by GMFCS and FA values was noticed in both right"* and left"** CST (" $r=-0.749, p<0.001$; *** $r=$ $-0.651, p<0.001)$ and CTR (" $r=-0.653, p<0.001$; *** $r$ $=-0.642, p<0.001)$ tracts. Significant positive correlation with MD was noticed in right CST $(r=0.216, p=$ 0.034) and right" and left"** CTR ( ${ }^{*} r=0.408, p=0.005$;

Table 2 Mean FA, MD values, fiber counts, and standard deviations measured in both CST and thalamic radiations in CP group and control group

\begin{tabular}{|c|c|c|c|c|c|c|}
\hline & \multicolumn{3}{|l|}{ Right } & \multicolumn{3}{|l|}{ Left } \\
\hline & Case & Control & Test of significance & Case & Control & Test of significance \\
\hline \multicolumn{7}{|l|}{ CST } \\
\hline FA & $0.395 \pm 0.045$ & $0.520 \pm 0.058$ & $\begin{array}{l}t=11.15 \\
p<0.001^{*}\end{array}$ & $0.379 \pm 0.09$ & $0.516 \pm 0.05$ & $\begin{array}{l}t=7.55 \\
p<0.001^{*}\end{array}$ \\
\hline MD & $0.997 \pm 0.165$ & $0.930 \pm 0.149$ & $\begin{array}{l}t=1.79 \\
p=0.08\end{array}$ & $0.962 \pm 0.234$ & $0.913 \pm 0.119$ & $\begin{array}{l}t=1.01 \\
p=0.313\end{array}$ \\
\hline Fiber count & $204.65 \pm 154.68$ & $1520.08 \pm 126.0$ & $\begin{array}{l}t=38.80 \\
p<0.001^{*}\end{array}$ & $182.83 \pm 141.59$ & $1480.38 \pm 160.06$ & $\begin{array}{l}t=38.50 \\
p<0.001^{*}\end{array}$ \\
\hline \multicolumn{7}{|l|}{ CTR } \\
\hline FA & $0.371 \pm 0.02$ & $0.469 \pm 0.09$ & $\begin{array}{l}t=6.15 \\
p<0.001 *\end{array}$ & $0.359 \pm 0.08$ & $0.458 \pm 0.03$ & $\begin{array}{l}t=4.33 \\
p<0.001^{*}\end{array}$ \\
\hline MD & $1.042 \pm 0.01$ & $0.923 \pm 0.02$ & $\begin{array}{l}t=27.44 \\
p<0.001^{*}\end{array}$ & $1.066 \pm 0.08$ & $0.925 \pm 0.05$ & $\begin{array}{l}t=5.92 \\
p<0.001^{*}\end{array}$ \\
\hline Fiber count & $773.84 \pm 250$ & $2022.11 \pm 310$ & $\begin{array}{l}t=14.39 \\
p=0.001^{*}\end{array}$ & $765 \pm 102$ & $1969 \pm 120.5$ & $\begin{array}{l}t=34.60 \\
p=0.001^{*}\end{array}$ \\
\hline
\end{tabular}

$R T$, right; $L T$, left; $C S T$, corticospinal tract; $C T R$, corticothalamic radiations; $M D$, mean diffusivity

A two-sample $t$-test analysis was performed to compare the results of ROI-based data between the CP and control groups. $p<0.001$ was applied to correct for multiple comparisons

*Bold text denotes significant between group differences following multiple comparisons correction with a level of significance determined at $p \leq 0.001$. Units for $\mathrm{MD}$ is $10-3 \mathrm{~mm}^{2} / \mathrm{s}$ whereas FA is dimensionless 
Table 3 Correlations between clinical motor grade and diffusion tensor imaging metrics (fractional anisotropy, mean diffusivity, and fiber count) of motor corticospinal and sensory corticothalamic tracts in cerebral palsy children (no $=70$ )

\begin{tabular}{llllll}
\hline & & Right CST & Left CST & Right CTR & Left CTR \\
\hline FA versus GMFCS & $r$ & $-\mathbf{0 . 7 4 9 ^ { * * }}$ & $-\mathbf{0 . 6 5 1 ^ { * * }}$ & $-\mathbf{0 . 6 5 3}^{* *}$ & $<.642^{* *}$ \\
MD versus GMFCS & $p$ & $<0.001$ & $<0.001$ & $<0.001$ & $\mathbf{0 . 4 0 8 ^ { * * }}$ \\
& $r$ & $\mathbf{0 . 2 1 6 ^ { * }}$ & 0.040 & 0.005 & $\mathbf{0 . 4 2 2 ^ { * * }}$ \\
Fibers count versus GMFCS & $p$ & 0.034 & 0.701 & $-\mathbf{0 . 7 0 8 ^ { * * }}$ & -0.001 \\
& $r$ & $-0.834^{* *}$ & $-0.815^{* *}$ & $<0.001$ & $<0.001$ \\
\hline
\end{tabular}

$r$ Spearman correlation coefficient

** Statistically significant

Range of correlation coefficient values $r:-1.00$ to -0.80 very strong negative; -0.79 to -0.60 strong negative; -0.59 to -0.40 moderate negative; -0.39 to 0.20 weak negative; -0.19 to -0.01 very weak negative; $r$ : 0.80 to 1.00 very strong positive; 0.60 to 0.79 strong positive; 0.40 to 0.59 moderate positive; 0.20 to 0.39 weak positive; 0.00 to 0.19 very weak positive

$*: r=0.422, p<0.001)$. Also, significant negative correlation among fiber count was noticed in both right* and left ${ }^{* * *}$ CST ( $r=-0.834, p<0.001 ;{ }^{* * *} r=-0.815, p<$ $0.001)$ and right" and left"** CTR (" $r=-0.708, p<0.001$; *** $r=-0.728, p<0.001)$ (Table 3).

\section{ROC curve analysis}

ROC curve analysis (Figs. 1 and 2) was carried out to assess the significance of cutoff values of FA distinguishing $\mathrm{CP}$ and the control group (Table 4), demonstrating right CST FA cutoff value was 0.449 with sensitivity $88.6 \%$, specificity $92.3 \%$, PPV 75\%, NPV 96.9\%, and accuracy $89.6 \%$, whereas left CST cutoff value was 0.472 with sensitivity $91.4 \%$, specificity $84.6 \%$, PPV $78.6 \%$, NPV $94.1 \%$, and accuracy $89.6 \%$.

For right CTRs, FA cutoff value was 0.432 with sensitivity $94.3 \%$, specificity $92.3 \%$, PPV $97.1 \%$, NPV $85.7 \%$, and accuracy $93.8 \%$, whereas for left CTRs cutoff value was 0.44 with sensitivity $97.1 \%$, specificity $76.9 \%$, PPV 91.9\%, NPV 90.9\%, and accuracy $91.7 \%$.

Also, there were significant differences among both studied groups in the measurement of MD values in different tracts (Table 5). For right CST, MD cutoff value was 0.903 with sensitivity $71.4 \%$, specificity $69.2 \%$, AUC $=0.638$, PPV 86.2\%, NPV 74.4\%, and accuracy 70.83\%; whereas for left CST cutoff value was 0.885 with sensitivity $91.4 \%$, specificity $53.8 \%$, AUC $=0.622$, PPV $80.6 \%$, NPV 41.2\%, and accuracy $66.7 \%$.

For right CTRs, MD cutoff value was 0.905 with sensitivity $70.1 \%$, specificity $68.6 \%$, PPV $45 \%$, NPV $85.7 \%$, and accuracy 69\%, whereas for left CTRs cutoff value was 0.909 with sensitivity $75.7 \%$, specificity $76.9 \%$, PPV $89.7 \%$, NPV 52.6\%, and accuracy $77 \%$.

\section{Discussion}

DTI has been essential to understanding the complexity and variability in brain injuries among CP children [9].

In this study, we assessed abnormalities on diffusion magnetic resonance imaging associated with $\mathrm{CP}$. CP group included $46(65.7 \%)$ preterm children and 24 (34.3\%) term children. The mean gestational weight was $2812.86 \pm 503.52$ (range, $1600-3800 \mathrm{~g}$ ). The gestational age in infants with $\mathrm{CP}$ was less than those in controls. The birth weight of children with CP $(2812.86 \pm 503.52)$ was significantly lower than birth weight among the control group $(3161.54 \pm 181.28)(p=0.001)$.

Such findings followed those of Schieve et al. [10], who reported that low birth weight-preterm and average birth weight-preterm were associated with $\mathrm{CP}$. Also, coping with the study done by McIntyre et al. [11], it described that the smaller the infant for gestational age and the lower the birthweight, the higher CP risk.

In this study, we found that 26 (37.1\%) of children suffer from perinatal hypoxia, whereas 36 (51.4\%) were admitted to the intensive care infant unit following delivery. These findings are in coping with a study done by Mcintyre et al. [11], which conclude that birth asphyxia was the powerful and the most reliable predisposing factor for $\mathrm{CP}$ and also with a study done by Ahlin et al. [12], which conclude that a neonatal

Table 4 The overall sensitivity, specificity, PPV, NPV, and AUC of FA values in CP and control group in CST and CTR

\begin{tabular}{lllllllll}
\hline Test result variable(s) & AUC & $\boldsymbol{p}$ & Cutoff point & Sensitivity (\%) & Specificity (\%) & PPV (\%) & NPV (\%) & Accuracy (\%) \\
\hline RT CST & 0.951 & $<0.001^{*}$ & 0.4490 & 88.6 & 92.3 & 75.0 & 96.9 & 89.6 \\
LT CST & 0.956 & $<0.001^{*}$ & 0.4725 & 91.4 & 84.6 & 78.6 & 94.1 & 89.6 \\
RT CTR & 0.965 & $<0.001^{*}$ & 0.432 & 94.3 & 92.3 & 97.1 & 85.7 & 93.8 \\
LT CTR & 0.965 & $<0.001^{*}$ & 0.440 & 97.1 & 76.9 & 91.9 & 90.9 & 91.7 \\
\hline
\end{tabular}

${ }^{*}$ means that the values are Statistically significant 
Table 5 The overall sensitivity, specificity, PPV, NPV, and AUC of MD values in CP and control group in CST and CTR

\begin{tabular}{lllllllll}
\hline Test result variable(s) & Area & $\boldsymbol{p}$ & Cutoff point & Sensitivity (\%) & Specificity (\%) & PPV (\%) & NPV (\%) & Accuracy (\%) \\
\hline RT CST & 0.638 & $0.038^{*}$ & 0.9035 & 71.4 & 69.2 & 86.2 & 47.4 & 70.83 \\
LT CST & 0.622 & 0.067 & 0.8855 & 71.4 & 53.8 & 80.6 & 41.2 & 66.7 \\
RT CTR & 0.758 & $0.001^{*}$ & 0.905 & 70.1 & 68.6 & 45.0 & 85.7 & 69.0 \\
LT CTR & 0.762 & $0.001^{*}$ & 0.909 & 75.7 & 76.9 & 89.7 & 52.6 & 77.0 \\
\hline
\end{tabular}

*means that the values are Statistically significant

encephalopathy and NICU admission are among the most substantial risk factors for $\mathrm{CP}$.

This study revealed that the most common brain lesions on conventional MRI were white matter lesions $(60 \%)$, followed by grey matter lesions $(8.6 \%)$, brain malformations (5.7\%), and lastly, postnatal events $(2.9 \%)$. Finally, $22.9 \%$ of studied patients had normal conventional MRI findings.

These findings were in agreement with those of Bax et al. [13], who observed that conventional MRI scans demonstrated that white matter injuries, such as PVL, were the most typical feature (42.5\%), followed by basal ganglia affection (12.8\%), cortical/ subcortical lesions (9.4\%), malformations (9.1\%), focal infarcts (7.4\%), and miscellaneous disorders (7.1\%). Only $11.7 \%$ of children showed normal MRI study.

Also, our results are coping with a study done by Franki et al. [14], which observed that most of the recorded lesions in CP cases are composed of white matter lesions $(66.9 \%$ of entire lesions) then grey matter affection (18.6\%) and brain malformations (4.3\%). Of note, postnatal lesions developed in 16 children (1\%) and the miscellaneous group (1\%), while they $(8.2 \%)$ show no insult on conventional MRI.

In contrast to conventional MRI, this study demonstrated a significant decrease in fractional anisotropy and fiber count and the CST on the affected side compared with control subjects. Also, there has been an increase in MD observed in this tract.

Such findings are consistent with those of Scheck et al. [15], who reported decreased FA and increased MD within the corticospinal pathway. These findings propose a reduction in CST integrity in comparison with typically developing children. Diffusion MRI and tractography research revealed additional proof of injury or perturbed initial development of this pathway.

Our results are also in the same line with those of Cho et al. [16] study who displayed that many DTI and DTT researches had demonstrated evidence of deficits in the CST of CP cases. The most expected recorded findings were the reduction in the number of fibers [17], reduction in FA, and increase in MD values [18].

These findings were in disagreement with those of Rha et al. [17], who reported that the numbers of fibers, but not FA or MD values, are lower in the CSTs in children with low gross motor function. This difference is due to the small sample size in a study done by Rha et al. [17], and the standard deviations were large, which was associated with more possible false-negative results. Besides, narrow age ranges of cases in which DTT was performed (between 7 months and 2.9 years) may be considered another explanation.

This study showed significant involvement (decrease in DTI fiber count) of the superior thalamic radiation (in severe cases) with atrophy of the ATR or posterior thalamic radiations (PTR) in most cases with a significant reduction in FA and increase in MD values.

These findings are coping with a study done by Trivedi et al. [7] in which a significant decrease in FA values was noted in both corticothalamic tracts moving from controls to GMFCS level V. Also, all the studied groups showed higher MD values in tract than the control group.

Also, our results agree with the study done by Arrigoni et al. [19], which discover that FA was significantly reduced in the thalamic radiations; therefore, such findings revealed the significance of sensory integration in the determinism of the multilevel damage in CP.

This result was different from that of Thomas et al. [20], whose research demonstrated significant involvement (decrease in DTI fiber count) of the superior thalamic radiation without a change in the ATR or PTR. Such difference between our results may be due to a small number of subjects included in Thomas et al. [20] with different age distribution.

This study also found a strong negative correlation of FA with a clinical motor grade in the corticothalamic and a corticospinal tract, which proposes that damaged corticothalamic tracts along with CST affection might have an essential role in the pathophysiology of motor dysfunction in CP cases.

Such findings are in the same line with a study done by Weinstein et al. [21], who described correlations between diffusion measures in motor and sensory tracts and motor functions.

This result was not coped with Lee et al. [22], who reported that FA mostly within CST demonstrated a more significant correlation with motor dysfunction in 


\section{ROC Curve}

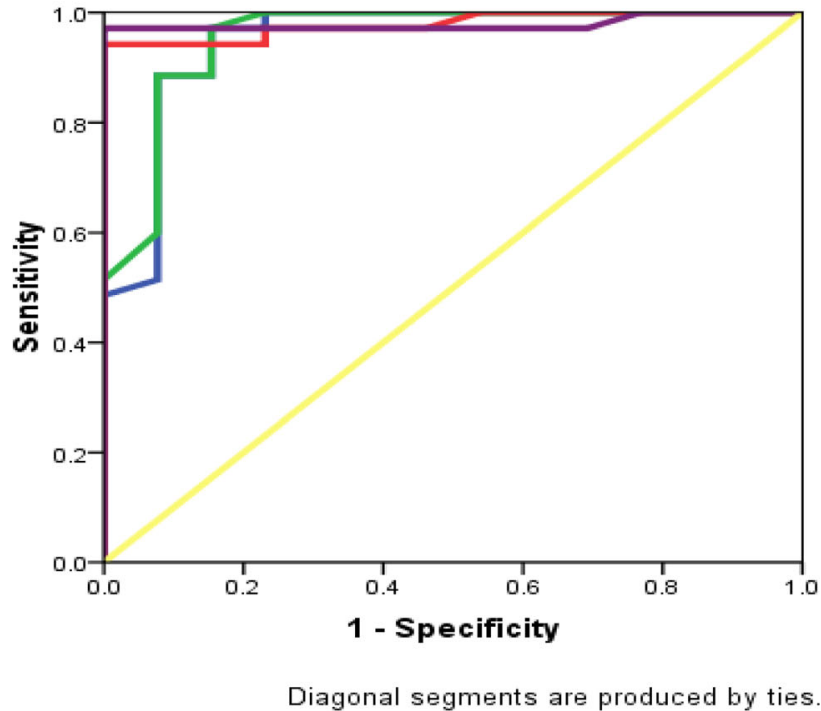

Source of the Curve

- Rt CST

LtCST

- Rt CTR

Reference line

Diagonal segments are produced by ties

Fig. 1 ROC curve application for determination of sensitivity and specificity of FA values in (CST and CTR) in CP diagnosis

comparison with thalamocortical pathways. The difference in these results may be due to different $\mathrm{CP}$ (diplegic) types included in this study.

This study also revealed a significant negative correlation between fibers count and different levels of GMFCS with corresponding differences detectable on DTT in corticospinal tracts and corticothalamic radiation (Fig. 3). This agrees with that of Yoshida et al. [23], who observed that in CP cases with different GMF levels, there were difference in fiber count in CST and thalamic radiations.

The higher MD values in motor and sensory tracts of the $\mathrm{CP}$ cases compared with the control group may be

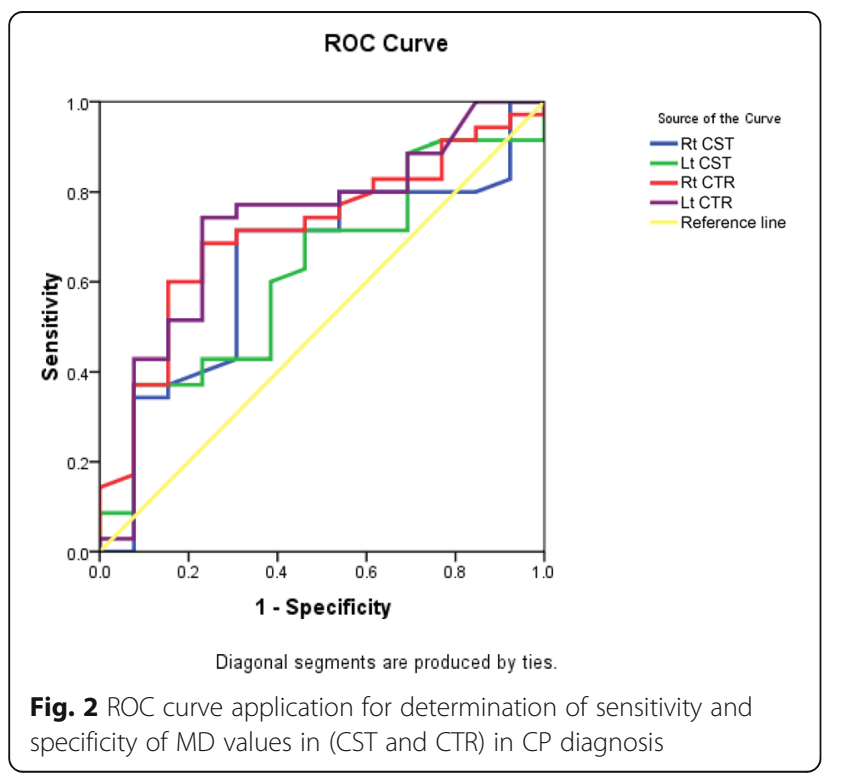

secondary to increased extracellular water contents due to marked gliosis and microscopic/macroscopic cystic changes that occurred in affected areas [24]. In this study, the MD values in $\mathrm{CP}$ cases were higher in both sensory corticothalamic and motor corticospinal tracts compared to controls, with a significant positive correlation noticed among GMFCS grade and MD values in sensory and motor pathways, which reflects an increase in extracellular water content and gliosis with higher GMFCS levels.

These findings are coping with those of Trivedi et al. [7], who observed the significant correlation between MD values noted in sensory corticothalamic and motor corticospinal tracts with clinical motor grades assessed by GMFCS.

This study revealed high sensitivity and specificity of DTI quantitative and qualitative measures in evaluating the WM tracts injury underlying $\mathrm{CP}$ disorders. The current results confirmed that FA values had high diagnostic values. Significant variations in FA values were observed among CP patients, and controls with detectable cutoff values of FA within CST were 0.472 (left) and 0.449 (right) tracts, and within CTR were 0.44 (left) and 0.432 (right) tracts. The significant predictive power of FA within the CTR had the highest value of accuracy (AUC 0.965), showing $97.1 \%$ sensitivity, $76.9 \%$ specificity, $90.9 \%$ negative, and $91.9 \%$ positive predictive values on the left side and $94.3 \%$ sensitivity, $92.3 \%$ specificity, $85.7 \%$ negative, and $97.1 \%$ positive predictive values on the right side.

Previous research done in 2008 by Murakami et al. [25] demonstrated that an FA threshold of 0.5 within the CST was efficient in distinguishing $\mathrm{CP}$ and non-CP groups, and a study done by Jiang et al. [26] also 


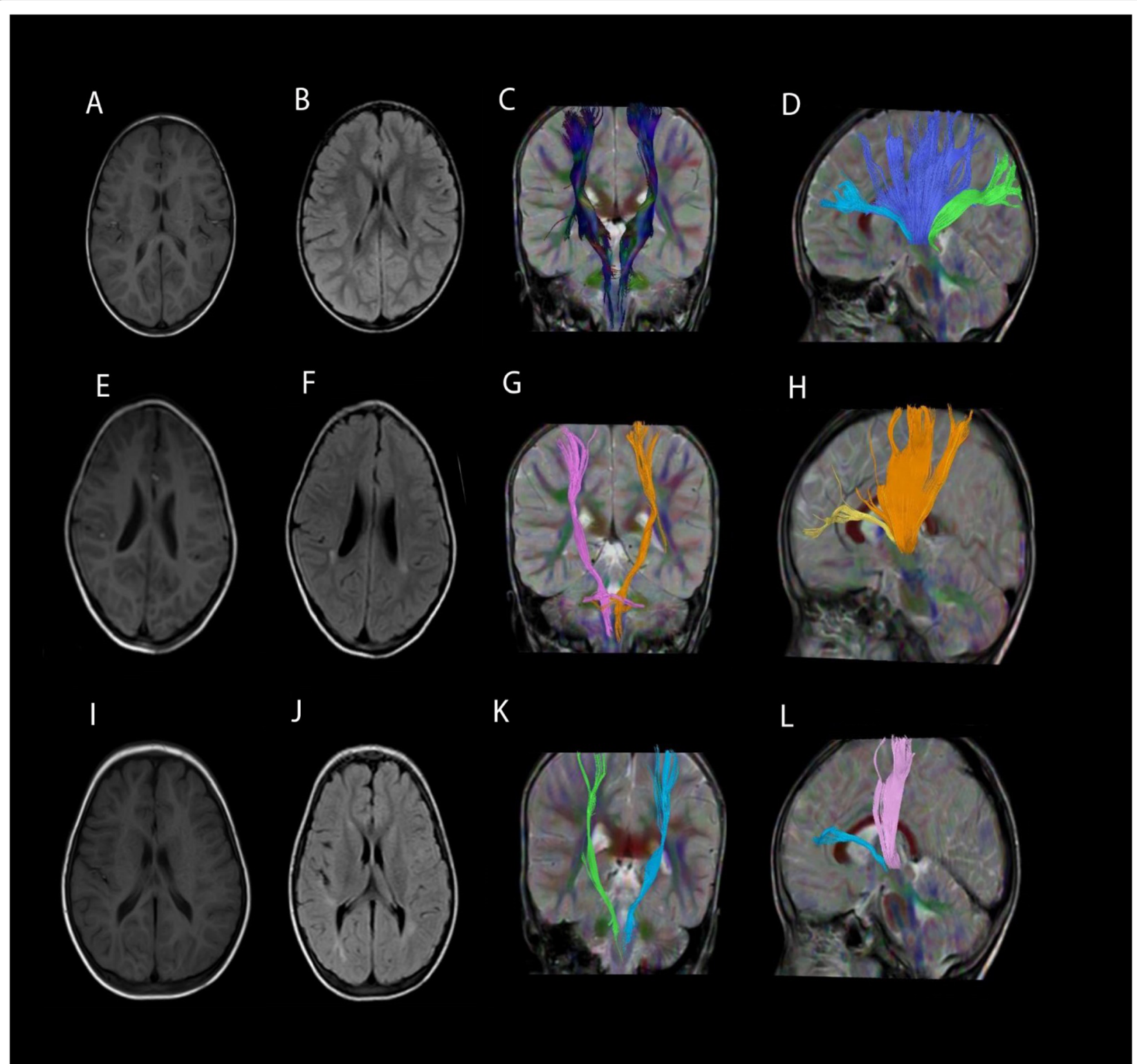

Fig. 3 Comparison between control, lower grades, and higher grades of GMFCS; control case 1st row (a, b) T1 WI and FLAIR images show normal study; c normal CST and $\mathbf{d}$ normal ATR (light blue colored), STR (blue colored), and PTR (green colored). Second row represents a case with mild motor affection (GMFCS II) with $\mathbf{e}, \mathbf{f} T 1 \mathrm{WI}$ and FLAIR images revealed mild PVL; $\mathbf{g}$ mild thinning of CST, $\mathbf{h}$ mild thinning of STR (Orange colored) and ATR (yellow colored) and absent PTR. Third row represents the case with severe motor affection with (GMFCS IV); i, j T1 WI and FLAI R images revealed mild PVL; $\mathbf{k}$ mild thinning of CST (more on right side) with I moderate tinning of STR (pink colored) and ATR (light blue colored) and absent PTR

revealed that FA threshold in CST for diagnosis of CP was 0.48 (left) and 0.49 (right).

This study suggests that sensory and motor tracts are affected in different degrees in CP cases, which lead to various clinical presentations in $\mathrm{CP}$ cases. These findings came following previous studies, which were carried out by Glenn et al. [27] and Thomas et al. [20], who suggest that both motor and sensory pathways are involved in CP patient with PVWI and a complex interplay of relative involvement of such pathways may define the terminal outcomes of such cases and not the degeneration of either of them alone.

Our results are in agreement with those of Scheck et al. [15], who demonstrated that marked impairment to descending motor and ascending sensorimotor tracts plays a role in the neuro-pathogenesis of $\mathrm{CP}$ and emphasize the significance of preservation of ascending sensorimotor networks in motor functions and also offer novel insights for the design of neurorehabilitation therapy. 
Also, they consist of Jiang et al. [26] and Mailleux et al. [28], who demonstrated clear associations among upper limb function and white matter integrity of the descending CST and ascending thalamocortical tracts in unilateral CP cases.

The current study has some limitations. First, the parenchymal damage in CP cases, with loss of WM volume and ventricular enlargement, might interfere with creating a standard DTI template among cases and control and affect DTI outcomes. However, the current study displayed moderate to marked ventricular enlargement in $8.5 \%$ of $\mathrm{CP}$ cases. The second limitation is the lack of dyskinetic and ataxic types of $\mathrm{CP}$.

\section{Conclusion}

This study revealed a significant correlation between DTI measures and tractography in motor and sensory tracts with clinical motor grading. This recommends that WM tracts from the somatosensory and the motor cortex display an essential role in terms of pathophysiology of motor disability in CP cases.

\section{Abbreviations}

ATR: Anterior thalamic radiation; CTR: Corticothalamic radiation; DTI: Diffusion tensor imaging; DTT: Diffusion tensor tractography; FA: Fractional anisotropy; GMFCS: Gross Motor Function Classification System; MD: Mean diffusivity; NICU: Neonatal intensive care unit; PTR: Posterior thalamic radiation; PWI: Periventricular white matter injury; ROI: Region of interest; STR: Superior thalamic radiation

\section{Acknowledgements}

Not applicable.

\section{Authors' contributions}

$\mathrm{NE}$ and $\mathrm{AM}$ designed the research. AM performed the research and wrote the manuscript. NE and GA analyzed the collected data. $A E, A M, G A$, and $K M$ revised data and manuscript. All authors read and approved the final manuscript.

\section{Funding}

Not applicable (no funding received for this study).

\section{Availability of data and materials}

Available on request with the corresponding author.

\section{Declarations}

\section{Ethics approval and consent to participate}

This study was approved by the research ethics committee of the Radiology Department of the Faculty of Medicine Mansoura University on 13/7/2017, Reference number of approval: MS/17.07.10. All patients included in this study gave a written informed consent to participate in the research.

\section{Consent for publication}

All patients included in this study gave a written informed consent to publish the data contained in this study.

\section{Competing interests}

The authors declare that they have no competing interests.

\section{Author details}

${ }^{1}$ Diagnostic and Interventional Radiology, Ministry of Health, Cairo, Egypt. ${ }^{2}$ Diagnostic and Interventional Radiology, Mansoura University, Mansoura, Egypt. ${ }^{3}$ Pediatrics and Neuropediatrics, Mansoura University, Mansoura, Egypt.
Received: 2 December 2020 Accepted: 14 March 2021

Published online: 24 March 2021

\section{References}

1. Arneson CL, Durkin MS, Benedict RE, Kirby RS, Yeargin-Allsopp M, Braun KVN, Doernberg NS (2009) Prevalence of cerebral palsy: autism and developmental disabilities monitoring network, three sites, United States, 2004. Disabil Health J 2(1):45-48. https://doi.org/10.1016/j.dhjo.2 008.08.001

2. Oskoui M, Coutinho F, Dykeman J, Jette N, Pringsheim T (2013) An update on the prevalence of cerebral palsy: a systematic review and meta-analysis. Dev Med Child Neurology 55(6):509-519. https://doi.org/10.1111/dmcn.12 080

3. Glader, L., Barkoudah, E., \& Armsby, C. (2018). Cereebral palsy: evaluation and diagnosis. In Post, T. W. (Ed.), UpToDate. Retrieved from https://www.uptoda te.com/contents/cerebral-palsy-evaluation-and-diagnosis

4. Deng W, Pleasure J, Pleasure D (2008) Progress in periventricular leukomalacia. Arch Neurol 65(10):1291-1295. https://doi.org/10.1001/a rchneur.65.10.1291

5. Berman Jl, Mukherjee P, Partridge SC, Miller SP, Ferriero DM, Barkovich AJ, Vigneron DB, Henry RG (2005) Quantitative diffusion tensor MRI fiber tractography of sensorimotor white matter development in premature infants. Neuroimage 27(4):862-871. https://doi.org/10.1016/j.neuroimage.2 005.05.018

6. Ashwal S, Russman BS, Blasco PA, Miller G, Sandler A, Shevell M, Stevenson $R$ (2004) Practice parameter: diagnostic assessment of the child with cerebral palsy: report of the Quality Standards Subcommittee of the American Academy of Neurology and the Practice Committee of the Child Neurology Society. Neurology 62(6):851-863. https://doi.org/10.1212/01. WNL.0000117981.35364.1B

7. Trivedi R, Agarwal S, Shah V, Goyel P, Paliwal VK, Rathore RK, Gupta RK (2010) Correlation of quantitative sensorimotor tractography with clinical grade of cerebral palsy. Neuroradiology 52(8):759-765. https://doi.org/10.1 007/s00234-010-0703-8

8. ElBeheiry AA, Elgamal MA, Ettaby AN, Omar TE, Badeib AO (2019) Can diffusion tensor imaging predict cerebral palsy in term neonates with hypoxic ischemic encephalopathy? Egypt J Radiol Nucl Med 50(1):66. https://doi.org/10.1186/s43055-019-0077-9

9. Hoon AH Jr, Stashinko EE, Nagae LM, Lin DD, Keller J, Bastian A et al (2009) Sensory and motor deficits in children with cerebral palsy born preterm correlate with diffusion tensor imaging abnormalities in thalamocortical pathways. Dev Med Child Neurol 51(9):697-704. https://doi.org/10.1111/j.14 69-8749.2009.03306.x

10. Schieve LA, Tian LH, Rankin K, Kogan MD, Yeargin-Allsopp M, Visser S, Rosenberg D (2016) Population impact of preterm birth and low birth weight on developmental disabilities in US children. Ann Epidemiol 26(4): 267-274. https://doi.org/10.1016/j.annepidem.2016.02.012

11. McIntyre S, Taitz D, Keogh J, Goldsmith S, Badawi N, Blair EVE (2013) A systematic review of risk factors for cerebral palsy in children born at term in developed countries. Dev Med Child Neurol 55(6):499-508. https://doi. org/10.1111/dmcn.12017

12. Ahlin K, Himmelmann K, Hagberg G, Kacerovsky M, Cobo T, Wennerholm UB, Jacobsson B (2013) Non-infectious risk factors for different types of cerebral palsy in term-born babies: a population-based, case-control study. BJOG 120(6):724-731. https://doi.org/10.1111/1471-0528.12164

13. Bax M, Tydeman C, Flodmark O (2006) Clinical and MRI correlates of cerebral palsy: the European Cerebral Palsy Study. Jama 296(13):1602-1608. https://doi.org/10.1001/jama.296.13.1602

14. Franki I, Mailleux L, Emsell L, Peedima ML, Fehrenbach A, Feys H, Ortibus E (2020) The relationship between neuroimaging and motor outcome in children with cerebral palsy: a systematic review-Part A. Structural imagin. Res Dev Disabil 100:103606. https://doi.org/10.1016/j.ridd.2020.103606

15. Scheck SM, Boyd RN, Rose SE (2012) New insights into the pathology of white matter tracts in cerebral palsy from diffusion magnetic resonance imaging: a systematic review. Dev Med Child Neurol 54(8):684-696. https:// doi.org/10.1111/j.1469-8749.2012.04332.x

16. Cho HK, Jang SH, Lee E, Kim SY, Kim S, Kwon YH, Son SM (2013) Diffusion tensor imaging-demonstrated differences between hemiplegic and diplegic cerebral palsy with symmetric periventricular leukomalacia. Am J Neuroradiol 34(3):650-654. https://doi.org/10.3174/ajnr.A3272 
17. Rha DW, Chang WH, Kim J, Sim EG, Park ES (2012) Comparing quantitative tractography metrics of motor and sensory pathways in children with periventricular leukomalacia and different levels of gross motor function. Neuroradiology 54(6):615-621. https://doi.org/10.1007/s00234-011-0996-2

18. Papadelis C, Kaye H, Shore B, Snyder B, Grant PE, Rotenberg A (2019) Maturation of corticospinal tracts in children with hemiplegic cerebral palsy assessed by diffusion tensor imaging and transcranial magnetic stimulation. Front Human Neurosci 13:254. https://doi.org/10.3389/fnhum.2019.00254

19. Arrigoni F, Peruzzo D, Gagliardi C, Maghini C, Colombo P, lammarrone FS, Pierpaoli C, Triulzi F, Turconi AC (2016) Whole-brain DTI assessment of white matter damage in children with bilateral cerebral palsy: evidence of involvement beyond the primary target of the anoxic insult. Am J Neuroradiol 37(7):1347-1353. https://doi.org/10.3174/ajnr.A4717

20. Thomas B, Eyssen M, Peeters R, Molenaers G, Van Hecke P, De Cock P, Sunaert S (2005) Quantitative diffusion tensor imaging in cerebral palsy due to periventricular white matter injury. Brain 128(11):2562-2577. https://doi. org/10.1093/brain/awh600

21. Weinstein M, Green D, Geva R, Schertz M, Fattal-Valevski A, Artzi M, Myers V, Shiran S, Gordon AM, Gross-Tsur V, Bashat DB (2014) Interhemispheric and intrahemispheric connectivity and manual skills in children with unilateral cerebral palsy. Brain Struct Funct 219(3):1025-1040. https://doi.org/10.1007/ s00429-013-0551-5

22. Lee JD, Park HJ, Park ES, Oh MK, Park B, Rha DW et al (2011) Motor pathway injury in patients with periventricular leucomalacia and spastic diplegia. Brain 134(4):1199-1210. https://doi.org/10.1093/brain/awr021

23. Yoshida S, Hayakawa K, Yamamoto A, Okano S, Kanda T, Yamori Y et al (2010) Quantitative diffusion tensor tractography of the motor and sensory tract in children with cerebral palsy. Dev Med Child Neurol 52(10):935-940. https://doi.org/10.1111/j.1469-8749.2010.03669.x

24. Rugg-Gunn FJ, Symms MR, Barker GJ, Greenwood R, Duncan JS (2001) Diffusion imaging shows abnormalities after blunt head trauma when conventional magnetic resonance imaging is normal. J Neurol Neurosurg Psychiatry 70(4):530-533. https://doi.org/10.1136/jnnp.70.4.530

25. Murakami A, Morimoto M, Yamada K, Kizu O, Nishimura A, Nishimura T, Sugimoto $T$ (2008) Fiber-tracking techniques can predict the degree of neurologic impairment for periventricular leukomalacia. Pediatrics 122(3): 500-506. https://doi.org/10.1542/peds.2007-2816

26. Jiang, H., Liu, H., Huang, T., Wu, L., Wu, F., Liu, C., Wang M., Jin C., Yang J. \& Li, X. (2020). Structural network performance for early diagnosis of spastic cerebral palsy in periventricular white matter injury. Brain Imaging Behav https://doi.org/https://doi.org/10.1007/s11682-020-00295-6.

27. Glenn OA, Ludeman NA, Berman JI, Wu YW, Lu Y, Bartha Al, Vigneron DB, Chung SW, Ferriero DM, Barkovich AJ, Henry RG (2007) Diffusion tensor MR imaging tractography of the pyramidal tracts correlates with clinical motor function in children with congenital hemiparesis. Am J Neuroradiol 28(9): 1796-1802. https://doi.org/10.3174/ajnr.A0676

28. Mailleux L, Franki I, Emsell L, Peedima ML, Fehrenbach A, Feys H, Ortibus E (2020) The relationship between neuroimaging and motor outcome in children with cerebral palsy: a systematic review_-Part B diffusion imaging and tractography. Res Dev Disabil 97:103569. https://doi.org/10.1016/.jidd.2 019.103569

\section{Publisher's Note}

Springer Nature remains neutral with regard to jurisdictional claims in published maps and institutional affiliations.

\section{Submit your manuscript to a SpringerOpen ${ }^{\circ}$ journal and benefit from:}

- Convenient online submission

- Rigorous peer review

- Open access: articles freely available online

- High visibility within the field

- Retaining the copyright to your article

Submit your next manuscript at $\boldsymbol{\nabla}$ springeropen.com 